\title{
In vitro ploidy manipulation in the genomics era
}

\author{
Zong-Ming Cheng $\cdot$ Schuyler S. Korban
}

Published online: 5 February 2011

(C) Springer Science+Business Media B.V. 2011

Angiosperm (flowering) plants are the most diverse group of seeds plants on Earth. Although the majority of extant plants are diploid, wherein somatic cells possess two sets of chromosomes, a significant number of plant species has evolved into polyploids, wherein somatic cells contain three or more sets of chromosomes. These polyploid plants frequently exhibit many distinctive morphological characteristics, such as larger-sized organs, as with flowers and fruits of many tetraploid plants, and seedless fruits of triploid plants. Developing polyploid plants has been of considerable interest for horticulturists, agronomists, and foresters. However, due to their multiple sets of chromosomes, determination of genetic control of traits of interest in polyploids has been much more challenging than that in diploid plants. Moreover, incidence of heterozygosity, even in diploid plants, can further complicate the elucidation of genetic inheritance of a given trait. Thus, developing haploids from polyploid plants or generating monoploids from diploid plants followed by their dihaploidization, via induced or natural doubling of chromosomes from monoploid plants, has become highly desirable for geneticists and plant breeders alike.

Over the last several decades, plant breeders, geneticists, and biotechnologists have been attempting to utilize various approaches towards production of haploids (mostly monoploids) and polyploids, primarily triploids and

Z.-M. Cheng ( $\bowtie)$

Department of Plant Sciences, University of Tennessee, Knoxville, TN 37996, USA

e-mail: zcheng@utk.edu

\section{S. S. Korban}

Department of Natural Resources and Environmental Sciences, University of Illinois at Urbana-Champaign, Urbana, IL 61801, USA tetraploids, both for genetic analysis studies and applied plant production. Although traditional breeding techniques such as hybridization and mutagenesis have played significant roles in generating desirable polyploid and haploid plants, various in vitro techniques have become routine in manipulating ploidy levels.

In the twenty-first century, the field of plant biology has rapidly moved into the genomics era, first signaled by completing the whole genome sequence of Arabidopsis in 2000. Upon further detailed analysis of this smallest genome of angiosperm species, it is revealed that Arabidopsis is a paleopolyploid plant, or an ancient polyploid, that has resulted from ancient whole genome duplications. This phenomenon has been confirmed to be widely spread among other higher plant species, based on several recently completed whole genome sequences. Therefore, this begs the question as to how these ancient polyploid plants have evolved and diverged into today's plants. This has become a hot research topic of investigation in recent years. One of the research tools that has become widely available to the scientific community is the artificial synthesis of various auto- and allo-polyploid plants followed by studies on how these synthesized polyploids have evolved and diverged over time. Likewise, haploid and dihaploid plants have increasingly been employed for studying allelism at the whole genome level.

Based on the above, we have assembled this special issue on "In vitro ploidy manipulation in the genomics era", which is divided into different sections. The first section covers reviews and updates of various techniques in generating haploid plants, including anther culture, isolated microspore culture, unfertilized ovary/ovule culture, and distant hybridization/chromosome elimination in cereal crops. The second section covers progress on various techniques in generating polyploids, including production 


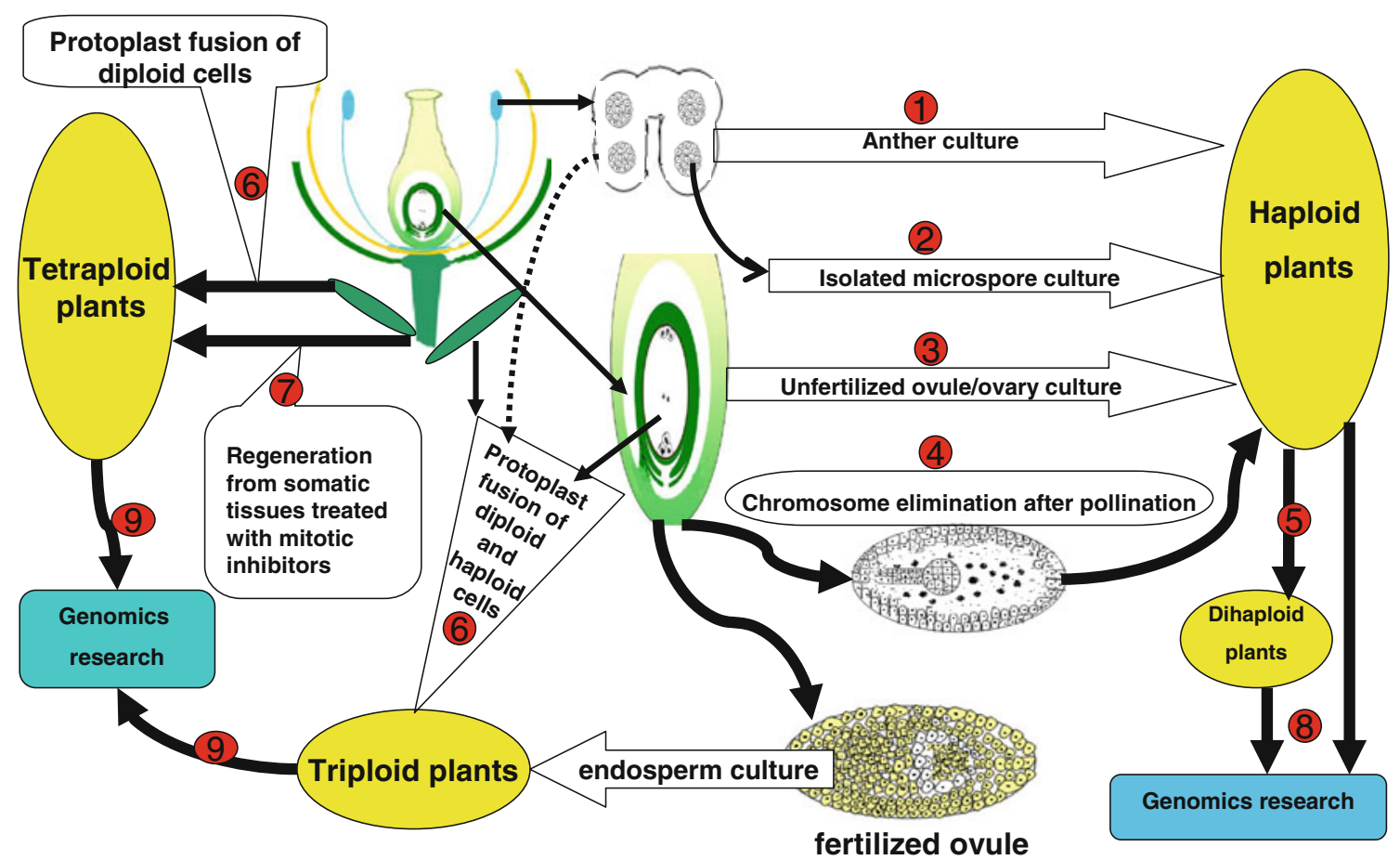

Fig. 1 Ploidy manipulation over different developmental stages and plant tissues/organs. Arrows point to various techniques used in these manipulations; while, circled numbers correspond to article number in this special issue

of triploids either by endosperm culture or by protoplast fusion (between diploid and haploid cells), production of autotetraploids by culture of various tissues with/without regeneration by treating target tissues with mitotic inhibitors, and auto- or allo-tetraploid production by protoplast fusion of diploid cells. Figure 1 illustrates these various haploid and polyploidy manipulation techniques in relation to the different plant developmental stages and organ/tissue types. The third section includes a couple of original research that has been conducted in this area. The fourth sections presents updates on how these haploids and polyploids can be used in genomics research. For example, Alison Ferrie and co-authors offer a thorough review of utilizing haploid and dihaploid for genomics research; while, Yang and colleagues offer an excellent review of recent progress in plant polyploid genomics.

Beyond summarizing the relevant fields of research, our main intention with this special issue is to further stimulate thinking beyond the current perspectives of various ploidy manipulation strategies or their application to traditional plant breeding. We hope that this special issue will inspire more innovative research by combining these relatively mature techniques with more current genomics research tools to advance our fundamental knowledge in plant biology that can also lead to novel practical innovations. 